\title{
ELASTIC PROPERTIES OF INVERSE POWER FLUIDS
}

\author{
A. C. BRAŃKA ${ }^{1 *}$ AND D. M. HEYES ${ }^{2 * *}$ \\ ${ }^{1}$ Institute of Molecular Physics, Polish Academy of Sciences \\ Smoluchowskiego 17, 60-179 Poznań, Poland \\ ${ }^{2}$ Chemistry Division, School of Biomedical and Molecular Sciences \\ University of Surrey, Guildford GU2 $7 X H, \quad U K$
}

(Rec. 7 October 2004)

\begin{abstract}
We investigated the consequences of variable potential softness on elastic properties, using the repulsive inverse power potential. With this potential the softness can be changed continuously from very soft to extremely steep or hard. An explicit formula for the equation of state is derived and discussed. It is shown how this formula can be exploited to determine the infinite frequency elastic properties of the inverse power fluid. Explicit formulae for the elastic constants, the high-frequency elastic moduli, the longitudinal- and transverse-wave velocities and Poisson's ratio are obtained. Their behaviour in the steeply repulsive limit is discussed. It is demonstrated that the softness directly determines the Poisson's ratio, and it is shown that in order to decrease the value of the Poisson's ratio a harder potential interaction must be applied.
\end{abstract}

\section{INTRODUCTION}

The softness of particles is an important characteristic which determines various physical properties of an assembly of interacting particles. It can be expressed in terms of the interaction between pairs of particles, defined usually in the form of an interaction potential, which is often "effective". Systems composed of particles of variable softness have attracted considerable attention in recent years. Much of this interest derives from the colloid and interface science communities, who can make particles of different softnesses for various applications. It is a convenient and useful distinguishing feature, as in the area of soft condensed matter the interacting units are often "fuzzy" objects. Also, the softness is an important parameter for granular materials.

The determination of various physical properties of an assembly of interacting particles directly from the interparticle forces is a non-trivial task and is still a largely unsolved problem, even in the case of model systems of particles interacting with a two-body spherically symmetric potentials. Such a potential is often the starting point for the description of the basic physical system properties of real systems. Among the different model interparticle potentials, the inverse-power or soft-sphere potential form seems to be particularly suitable for investigating the role of particle softness. First of all it has the simple analytic form,

$$
u(r)=\varepsilon\left(\frac{\sigma}{r}\right)^{n}
$$

\footnotetext{
*e-mail: branka@ifmpan.poznan.pl; **e-mail: d.heyes@surrey.ac.uk
} 
where $r$ is the separation between two particles, $\sigma$ is the particle diameter, $\varepsilon$ sets the energy scale and $n$ is a parameter determining the potential steepness (the softness is $\sim n^{-1}$ ). Thus, the single parameter, $n$, changed continuously can cover a wide spectrum of practically important systems from the very soft to the extremely hard, i.e., from the long-ranged Coulomb interaction $(n=1)$ to the hard sphere system $(n \rightarrow \infty)$. Apart from its simplicity the inverse-power potential has many features that make this potential attractive as a model system.

From its definition in (1), we see that the inverse power potential is a self-similar function, which mixes the energy and distance scales. It can be simply represented as $r^{*_{-} n}$, where $r^{*}=r / \sigma \varepsilon^{1 / n}$. A quite exceptional feature of the inverse power system is that the configurational properties of such system do not depend upon the density and temperature separately but upon a particular dimensionless combination [1]. In effect the properties computed along one isotherm (or isochore) are sufficient to determine the entire phase behaviour.

Another useful feature of the inverse power potential is the mutual relation between some basic physical properties of a system of such particles. For example the pressure and mechanical properties are trivially related to the interaction energy per particle.

Recently we have shown that the self-similar nature of the inverse-power potential is also reflected in the local behaviour of the structural properties [2]. It was shown that the cavity or indirect correlation function can locally (in the particle contact region) be well represented by a power or algebraic function, and such local scaling relation is obeyed for at least $n \geq 18$. As a result, the equation of state of the inverse power system has been determined for the entire fluid phase (as the potential is purely repulsive and no liquid-gas transition exists) for $n \geq 18$.

The self-similarity of the soft-sphere potential also has the consequence that uniform scaling of the particle co-ordinates does not produce changes in the potential energy landscape [3]. Soft sphere potential systems are useful for perturbation theories of fluids where they play the role of a reference system. A detailed knowledge of the properties of the inverse-power system would facilitate perturbation treatments of more complex molecular systems. Also, there is growing interest in colloidal and granular systems, whose particles experience a wide range of repulsive interactions. These can be modeled reasonably well by the inverse power. Purely repulsive $r^{-n}$ potential systems have been investigated by several authors particularly for $n=12$ and $n=6$ [4-8].

Depending on the softness, the $r^{-n}$ system freezes either into the $f c c$ or $b c c$ crystal structure, and this interesting softness-driven transition has been extensively studied $[9,4,5,10]$.

In this work we continue our investigations of the physical properties of the inverse-power system. In particular we concentrate on its elastic properties in the fluid phase. The work is part of our investigations on the effects of particle softness on elastic, viscoelastic and rehological properties of molecular and colloid systems.

A general equation of state formula and its explicit form is discussed in Sec. 2 and Sec. 3. The explicit forms of elastic properties are derived in Sec. 4 and conclusions are in Sec. 5. 


\section{THEORY}

Because of the scaling properties of the $r^{-n}$ potential the excess thermodynamic properties can be expressed in terms of a temperature-scaled density $\tilde{\rho}=(\beta \varepsilon)^{3 / n} \rho \sigma^{3}$, where $\rho=N / V$ is the number density and $\beta=1 / k_{B} T$ with $k_{B}$ Boltzmann's constant. Thus, it is possible and convenient to perform calculations in the following reduced units: $\tilde{r}=r \sigma^{-1}(\beta \varepsilon)^{-1 / n}, \quad \tilde{u}=\beta u=\tilde{r}^{-n}$. and $\tilde{P}=P \varepsilon^{-1} \sigma^{3}(\beta \varepsilon)^{1+3 / n}$. Also a temperature-scaled packing fraction, $\zeta=\pi \tilde{\rho} / 6$, is used to characterise density of the system.

As a consequence the equation of state and energy per particle can be expressed in a useful form,

$$
\begin{gathered}
\frac{\tilde{P}}{\tilde{\rho}}=1+4 \tilde{\zeta} \int d \tilde{r} \tilde{r}^{3} \frac{d \tilde{u}}{d \tilde{r}} g(\tilde{r}), \\
\langle\tilde{u}\rangle=12 \tilde{\zeta} \int d \tilde{r} \tilde{r}^{2} \tilde{u} g(\tilde{r}) .
\end{gathered}
$$

From the above formulae we can deduce a well known simple relation $\tilde{P} / \tilde{\rho}-1 \equiv Z=n<\tilde{u}>/ 3$ [11]. For the $r^{-n}$ system several other basic physical properties can be expressed in terms of $\langle u\rangle$ or the integral $Y=\int d \tilde{r} \tilde{r}^{2} \tilde{u} g(\tilde{r})$. Thus, if we could calculate $Y(\xi, n)$ we could determine a large part of the physical behaviour of the $r^{-n}$ system.

In what follows we omit the tilde, so keep in mind that we are dealing with temperaturescaled quantities.

In a previous work [2] we have shown that the cavity or indirect correlation function, $y(r)=g(r) \exp (\beta u)$, can in the particle contact region be well represented by the power or algebraic function $\exp (A) r^{-c}$, where $A$ and $C$ are functions of $n$ and $\zeta$ and $g(r)$ is the radial distribution function. With this analytic form of the cavity function the key integral $\Upsilon$ can be explicitly calculated (at least for $n \geq 18$ ) and the general formula for (the equation of state) energy of the inverse power fluid is,

$$
Z=4 \zeta \Gamma(1+(C-3) / n) e^{A} .
$$

In the above equation, $\Gamma$ denotes the Gamma function. In the limit $n \rightarrow \infty$ both the $A(\zeta, n)$ and $C(\zeta, n)$ functions have regular (smooth, monotonic, and finite) forms which allow us to express the quantity in equation (4) in terms of the hard-sphere equation of state, $P_{H S}$, namely,

$$
Z(\zeta, n)=Z_{H S}(\zeta) \Gamma(1+(C-3) / n) e^{\delta A}
$$

In the above equation, $\left.Z_{H S}=P_{H S}\right\rfloor-1$, and $\delta A=A-A_{H S}$, where $A_{H S}$ stands for $A(\zeta, n \rightarrow \infty)$. For the $Z_{H S}$, there are several accurate analytical representations available [12], for example, the established Carnahan and Starling [13], or that of Kolafa [14] given below,

$$
Z_{H S(\zeta)}=\frac{1+\zeta+\zeta^{2}-2\left(\zeta^{3}+\zeta^{4}\right) / 3}{(1-\zeta)^{3}}-1
$$


In this situation, it is sufficient to concentrate on the $\delta A$ and $C$ functions which determine the remaining, soft part of equation (5). These functions have been determined in our previous works [2, 15], where it was found that the $\mathrm{C}$ function has the useful feature that it is rather weakly dependent on $n$, and for larger $n$ it can be well represented by its limiting form, $C(\zeta, 0)$. This limiting form, $C(\zeta, n \rightarrow \infty)$ is directly related to the contact values of the derivatives of the hard sphere radial distribution function $-g\left(\sigma^{+}\right)^{-1} d g(r) /\left.d r\right|_{r=\sigma^{+}}$for which several accurate analytical representations has been proposed. Recent computer simulations demonstrate that the following equation can be derived by [16],

$$
C(\zeta, 0)=\frac{9 \zeta(1+\zeta)\left(1+\zeta / 4+\zeta^{2}+a \zeta^{3}\right)}{2-\zeta}
$$

which represents the simulation data particularly well (see Fig. 2 in [17]) if $a=3$ is used. With this limiting function a general representation of $C(\zeta, \varepsilon)$ is,

$$
C(\zeta, \varepsilon)=C(\zeta, 0)\left(1+\sum_{k=1}^{m} a_{k}(\zeta \varepsilon)^{k}\right),
$$

In the above formula the first four terms in the sum (i.e., $m=4$ ) are sufficient to represent the simulation data well, practically down to $n=12$, in the whole density range of the fluid phase.

For larger $n$ and $\zeta<0.3$ the linear term $(k=1)$ is sufficient. It is worth noting that the C function occurs in the equation of state formula (5), indirectly, via the argument $(C-3) / n$ of the Gamma function, whose value is close to unity. Thus it is not essential to know the particular form of the $n$-dependence of $C$. In our calculations we have used the following coefficients, $a 1=36, a 2=1400, a 3=12000, a 4=62986$.

The $n$ dependence of the $\delta A$ function is more significant than that of the $C$ function. For any $n, \delta A$ is a smooth, fairly regular function of $\zeta$, and $\delta A(n, 0)=0$ thus, it is plausible to consider it to be a polynomial,

$$
\delta A(\zeta, \varepsilon)=W_{1}(\varepsilon) \zeta+W_{2}(\varepsilon) \zeta^{2}+W_{3}(\varepsilon) \zeta^{3}+\ldots
$$

The explicit form of the $W$-functions can be established by exploiting the available simulation data and, for the low density region, the virial coefficients are available for the inverse power system. Expanding the right had side of Eq. (5) up to $\xi^{3}$ and comparing with the virial expansion $Z=B_{2} \zeta+B_{3} \zeta^{2}+B_{4} \zeta^{3}+\ldots$ one can write down the $W_{1}$ and $W_{2}$ functions in terms of the first four virial coefficients and the $C$-function, without involving any adjustable parameters or simulation data [15]. The computed $W_{1}$ and $W_{2}$ functions are smooth, monotonically increasing functions and are very well represented by the following polynomials:

$$
W_{1}=w_{10} \varepsilon+w_{11} \varepsilon^{2}+w_{12} \varepsilon^{3},
$$




$$
W_{2}=w_{20} \varepsilon+w_{21} \varepsilon^{2}+w_{22} \varepsilon^{3}+w_{23} \varepsilon^{4}
$$

where $w_{10}=6.9219, w_{11}=22.6574, w_{12}=-10.1574$ and $w_{20}=10.4067, w_{21}=4.4269$, $w_{22}=961.6914, w_{23}=-2678.5$. The above more convenient representation of the $W_{1}$ and $W_{2}$ coefficients is utilised in the further considerations.

The second order approximation for $\delta A$ yields the equation of state formula,

$$
Z_{2}(\zeta, n)=Z_{H S}(\zeta) \Gamma(1+(C-3) / n) e^{W_{1} \zeta+W_{2} \zeta^{2}}
$$

which describes almost completely the thermodynamic properties of the steeply repulsive inverse power fluid. It represents accurately the systems with $n>18$ up to $\zeta \sim 0.4$, and produces exactly in the low density expansion the first three virial coefficients (i.e., $B 2, \mathbb{B} 3$, B4).

In order to get a more accurate description of softer systems and/or systems in the dense fluid region (close to the freezing density) the function, $\delta A$ needs to be represented by a higher order polynomial and the additional $W$-coefficients can be determined by exploiting the available simulation data. We have found that they can be well represented by $W_{3} \xi^{3}+$ $+W_{4} \xi^{4}+W_{5} \xi^{\xi}$ where,

$$
\begin{gathered}
W_{3}=6.950(10 \varepsilon)^{3}, \\
W_{4}=\sum_{k=1}^{5} w_{4 k} \varepsilon^{k} \\
W_{5}=\sum_{k=1}^{5} w_{5 k} \varepsilon^{k}
\end{gathered}
$$

and the $w_{4 k}$ coefficients are $0,-9000,225600,-1866200,4150900$ and the $w_{5 k}$ coefficients are $-20,20500,-380400,2212200,-1471100$.

Finally we have obtained an explicit equation of state formula (5) composed of the hard sphere part $Z_{H S}(\zeta)(6)$, the soft $\Gamma$-part, (8), and the soft $\delta A$-part $(10,11,13-15)$ which gives an accurate representation of the equation of state of the inverse power fluid and correctly describes its low-density behaviour.

\section{3. $Z(C, e)$ SURFACE}

The general formula for $Z(\zeta, \varepsilon)$ in Eq. (5) is a simple product of three distinct and well defined parts: (i) the hard sphere equation of state, in this work represented by the Kolafa equation, (ii) the Gamma function representing the contribution of the $C$ function, and (iii) the exponential of $\delta A$, representing the contribution of the $A$ function. The resulting $Z(\zeta, \varepsilon)$ surface is drawn in Fig. 1. The bold line at $Z(\zeta, \varepsilon)$ is the freezing curve determined by Agrawal and Kofke [10], who investigated a very broad range of the softness parameter applying the Gibbs-Duhem method. Drawing the $Z(\zeta, \varepsilon)$ above as well as below the freezing line helps us 
visualise some of the general features of the thermodynamically stable fluid phase. From Fig. 1 it is clearly visible that $Z(\zeta, \varepsilon)$ is not a monotonic surface, but contains a fold or is composed of two well defined regions. In one region $(\partial Z / \partial \varepsilon)_{\zeta}$ is positive and in the second one it is negative. We may also say that there are two regions, characterised respectively by a positive and negative softness "compressibility", which we can define via the $\chi=(\partial Z / \partial \varepsilon)_{\zeta}^{-1}$ In other words to increase pressure (at a given packing fraction) in the one region we have to make the system softer, and to increase pressure in the second region we have to make system interaction steeper (i.e., increase $n$ ).

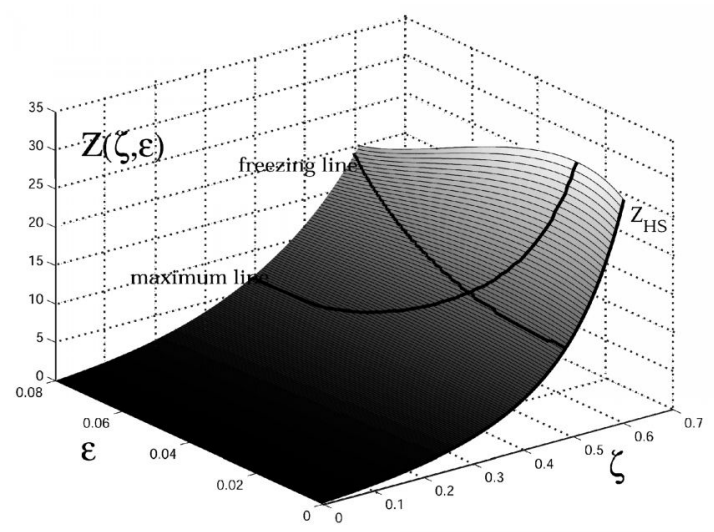

Fig. 1. The equation of state surface, $Z(\zeta, \varepsilon)$, of the inverse-power fluid, Eq. (5). $\zeta$ is the packing fraction and $\mathcal{E}=1 / n$ is the softness parameter. In the figure the freezing curve determined by Agrawal and Kofke [10] is drawn. Note that the surface is composed of two regions defined by positive and negative softness "compressibility". The bold line is the demarcation or maximum line, where the condition $(\partial Z / \partial \mathcal{E})_{\zeta}=0$ is obeyed

The demarcation line can be determine (at least numerically) from the condition $(\partial \delta A / \partial \varepsilon)_{\zeta}=-(\partial \log (\Gamma) / \partial \varepsilon)_{\zeta}$ which is the maximum condition $(\partial Z / \partial \varepsilon)_{\zeta}=0$ for $Z(\zeta=$ const, $\varepsilon)$. Let $\zeta^{M}$ be the value of this maximum backing fraction for a given $\varepsilon$. The demarcation line between these two regions is a maximum curve on the $Z\left(\zeta^{M}(\varepsilon)\right.$ ) surface (see Fig. 1). The maximum curve is fairly flat for soft systems and rises up considerably for smaller $\varepsilon$. As may be seen on the figure, for low packing fractions $(\zeta<0.3663) \chi$ is positive for all the considered $\varepsilon$. (For the $\varepsilon \leq 0.08$ considered here; for $\varepsilon=1 / 12$ the corresponding limiting density is estimated to be 0.3633 .) For increasing packing fraction a negative $\chi$ region starts to emerge, and for increasing $\varepsilon$ occupies more of the fluid range. In fact for a value of $\varepsilon=0.03$ (or $32<n<33$ ) it extends right up to freezing point.

For the low packing fractions, the pressure increases as the interaction gets softer, suggesting that it is the decay rate of the potential at long distances $(r>\sigma)$ that dominates the pressure in this part of the $\zeta$, $\varepsilon$ plane. Clearly as the potential becomes more long ranged it includes more of the neighbours, which can contribute to the pressure. In contrast, at high packing fraction, the number of interactions is already quite large (for not too larger), and it is the short range part of the potential $(r<\sigma)$ which apparently dominates any trend. As the potential gets longer ranged, the contribution to the pressure from close neighbours diminishes. Hence the pressure decreases as $\varepsilon$ increases, below some critical $\varepsilon$ at a given $\zeta$. For very 
large $n$, even at higher packing fractions, the pressure decreases as the interaction becomes stiffer, because of the relatively few interacting neighbours.

In the next Section we discuss how we can build on this treatment of the equation of state of the soft sphere fluid to derive relatively simple formulae for the various elastic constants at infinite frequency and related properties.

\section{THE ELASTIC PROPERTIES}

Several macroscopic properties of liquids can be written in terms of two important integrals $I_{1}$ and $I_{2}[18,19]$ :

$$
\begin{aligned}
& I_{1}=12 \zeta \int r^{3} g(r) \frac{d u}{d r} d r \\
& I_{2}=12 \zeta \int r^{4} g(r) \frac{d u^{2}}{d r^{2}} d r
\end{aligned}
$$

For example the above equation of state or compressibility factor is simply, $Z=-I_{1} / 3$. The second-order elastic constants in an isotropic medium can be written as

$$
\begin{gathered}
C_{11}=3+2 I_{1} / 5+I_{2} / 5, \\
C_{12}=1-2 I_{1} / 5+I_{2} / 15, \\
C_{44}=1+4 I_{1} / 15+I_{2} / 15 .
\end{gathered}
$$

These formulae apply in the limit of infinite frequency [20]. In deriving the above relations, central forces have been assumed. This ensures that the expressions satisfy the Cauchy relation $C_{44}=\left(C_{11}-C_{12}\right) / 2$, which reduces the number of independent constants to two. (The elastic constants are given in units of $\rho k_{B} T$ ). The high-frequency elastic moduli (also in the $\rho k_{B} T$ units) can similarly be written in terms of $I_{1}$ and $I_{2}$, as follows, for the infinite frequency elastic shear modulus,

$$
G_{\infty}=C_{44}-\rho / 15,
$$

for the high-frequency bulk modulus,

$$
K_{\infty}=\left(6+I_{1}+I_{2}\right) / 9
$$

for the high-frequency dilation modulus,

$$
M_{\infty}=K_{\infty}+4 G_{\infty} / 3
$$

The (instantaneous) bulk modulus $B$, is given by 


$$
B=\frac{C_{11}+2 C_{12}}{3}=\frac{5}{3}-\frac{2}{9} I_{1}+\frac{1}{9} I_{2}
$$

Takeno and Goda [21] have shown that the longitudinal- and transverse-wave velocities are related to $g(r), d u / d r$ and $d^{2} n / d r^{2}$ and can be written as,

$$
\begin{gathered}
v_{L}=\left[S\left(3 I_{2}+4 I_{1}\right)\right]^{1 / 2}, \\
v_{T}=\left[S\left(I_{2}+4 I_{1}\right)\right]^{1 / 2},
\end{gathered}
$$

where $S$ is a constant. Consequently, the Poission's ratio,

$$
v=\frac{v_{L}^{2}-2 v_{T}^{2}}{2\left(v_{l}^{2}-v_{T}^{2}\right)},
$$

which can also be written in terms of the $I_{1}$ and $I_{2}$ integrals, [19].

Thus, the main elastic properties can be obtained knowing the $I_{1}$ and $I_{2}$ integrals. At this point we note a useful feature of the inverse-power potential, namely for this potential $I_{2}=-(n+1) / I_{1}=3(n+1) Z$. In other words, our knowledge of $Z(\xi, n)$ for the soft-sphere fluid (see Sec. 2 and 3) is sufficient to obtain explicit formulae for the main elastic properties of the inverse-power fluid. Consequently we have the fairly extraordinary and unique situation in which explicit formulae for elastic properties are available:

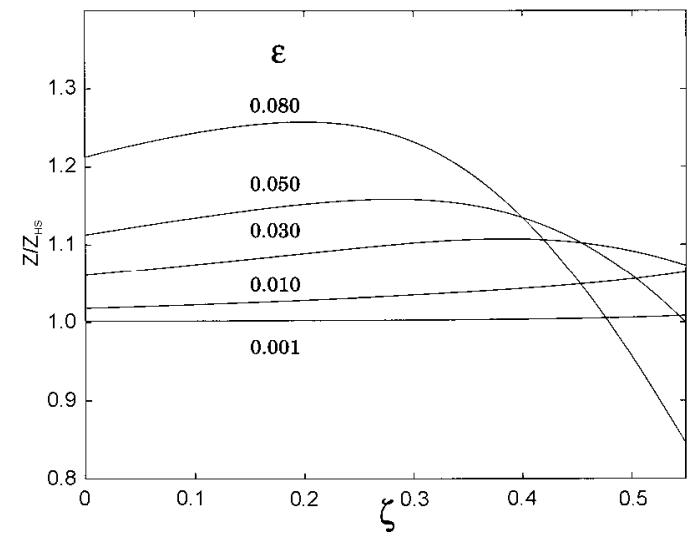

Fig. 2. The ratio $Z / Z_{H S}$ versus the packing fraction for a few different softness

$$
\begin{aligned}
& C_{11}=3+(3 n+1) Z / 5, \\
& C_{12}=1+(n+7) Z / 5, \\
& C_{44}=1+(n-3) Z / 5 .
\end{aligned}
$$


From the above formulae we see immediately that $C_{11}>C_{12}>C_{44}$, and this condition is obeyed for any $n$. As is shown in Fig. 2, for the softer interactions the details of the $Z$-surface (i.e., its $\zeta, n$-dependence) are significant and should not be neglected. For harder or steeper systems $(n>1)$ the term linear in «becomes dominant $\left(Z / Z_{H S} \rightarrow 1\right)$ and $C_{44} \approx C_{12} \approx C_{44} / 3 \rightarrow$ $n Z_{H S} / 5$. We have similar behaviour for the high-frequency modulus, $K_{\infty}=2+n Z, G_{\infty}=14 / 15+$ $+(n-3) Z / 5=C_{44}-1 / 15, M_{20}=3^{19} / 45+4(n-3) Z / 15$.

The longitudinal- and transverse-wave velocities are

$$
\begin{aligned}
& v_{L}=[3 S(3 n-1) Z]^{1 / 2}, \\
& v_{T}=[3 S(n-3) Z]^{1 / 2},
\end{aligned}
$$

which means that both velocities increase with system steepness as $\sqrt{ } n$. Furthermore, as expected, the longitudinal-wave velocity is greater than the transverse-wave velocity, and $v_{L}=v_{f}[1+2(n+1) /(n-3)]^{1 / 2}$ at any $\xi$ and $n$. Thus, for the harder system $v_{L} \cong v_{T}[3+8 / n]^{1 / 2}$ or goes to $v_{L} \cong \sqrt{3} v_{T}$. It is interesting to observe that the ratio $v_{L} / v_{T}$, is actually at its minimum in the hard sphere limit. In order to increase it we have to make the system softer (i.e., decrease the $n$-value). Finally, the Poission's ratio is

$$
v=\frac{n+5}{4(n+1)}=\frac{1}{4}+\frac{19}{16 n}+\ldots .
$$

Similarly, its minimum is in the hard sphere limit and we can also increase its value by making the system softer.

\section{CONCLUSIONS}

In this work the role of potential softness on the macroscopic properties of an ensemble of interacting particles was considered. It was pointed out that a system of inverse power particles exhibits several unique features, which make it a useful model system. This system exhibits a local scaling of structural properties which in turn allows us to obtain a general equation of state formula, $Z(\zeta, n)$. Incorporating the first few virial coefficients and computer simulation data we have established its explicit form. It was shown how knowledge of $Z(\zeta, n)$ allows us to obtain explicit formulae for the main elastic properties of the in verse-power fluid. For the first time the softness (i.e., $1 / n$ ) dependence of the elastic constants, the elastic moduli, the wave velocities and the Poisson's ratio have been established. The behaviour of elastic constants and the high-frequency modulus is driven by the $n Z(\zeta, n)$ term which can be approximated by the $n Z_{H S}$ in the steeply repulsive limit. For less steep interactions the details of the dependence of the Z-surface are important and cannot be neglected, particularly for the dense fluid region. For lower packing fractions $(\zeta<0.3)$ the simpler and fairly accurate low density equation of state formula (12) can be used. The Poisson's ratio decreases with increasing steepness as $1 / n$ and reaches its minimum value 0.25 in the hard sphere limit. 
Similarly the ratio of the longitudinal- to transverse-wave velocities is minimum, $\sqrt{3}$ in this limit. Our considerations demonstrate that one possible mechanism to increase the Poisson's ratio is enhance the softness of the interaction between particles. As a result of our studies the main elastic properties of the inverse power fluid have been determined for an appreciable range of the steepness parameter, $n$. It can be used as a basis for understanding better other molecular systems with a "tunable" softness.

\section{Acknowledgments}

$\mathrm{ACB}$ and DMH thank the Royal Society (London) and the Polish Academy of Sciences for funding this collaboration. The work has been partially supported by the Polish Committee for Scientific Research (KBN) grant No. 4T11F01023. DMH thanks the Engineering and Physical Sciences Research Council of Great Britain (EPSRC) for funding work stations used to carry out the computational aspects of this work.

\section{References}

[1] W. G. Hoover, S. G. Gray, and K. W. Johnson, J. Chem. Phys. 55, 1128 (1971).

[2] A. C. Branka and D. M. Heyes, Molec. Phys. 102, 2049 (2004).

[3] E. LA Nave, F. Sciortino, P. Tartaglia, M. S. Shell, and P. G. Debenedetti, Phvs. Rev. E 68, 032103 (2003).

[4] B. B. Laird and A. D. J. Haymet, Molec. Phys. 75, 71 (1992).

[5] R. Agrawal and A. D. Kofke, Phys. Rev. Lett. 74, 122 (1995).

[6] J.-P. Hansen and J. J. Weis, Molec. Phys. 23, 853 (1972).

[7] H. S. Kang, Ch. S. Lee, and T. Ree, J. Chem. Phys. 82, 414 (1984).

[8] B. B. Laird and A. D. J. Havmet, J. Chem. Phvs. 91, 3638 (1989).

[9] W. G. Hoover, J. Chem. Phys. 56, 2207 (1972).

[10] R. Agrawal and A. D. Kofke, Molec. Phys. 85, 23 (1995).

[11] D. M. Heyes and J. G. Powles, Mol. Phys. 95, 259 (1998).

[12] M. Miandehy and H. Modarress, J. Chem. Phys. 119, 2716 (2003).

[13] N. F. Carnahan and K. E. Starling, J. Chem. p'hys. 51, 635 (1969).

[14] T. Boublik and I. Nezbeda, Coll. Czech. Chem. Commun. 51, 2301 (1986).

[15] A. C. Branka and D. M. Heyes, in preparation.

[16] M. F. del Rio and A. L. Benavides, Mol. Phys. 72, 307 (1991).

[17] H. Sigurgeirsson and D. M. Heves, Mol. Phvs. 101, 469 (2003).

[18] P. Schofield, Proc. Phys. Soc. 88, 149 (1966).

[19] R. V. Gopala Rao and R. Venkatesh, Phys. Rev. B 39, 9467 (1989).

[20] S. Hess, M. Kröger, and H. Voigt, Physica A 250, 58 (1998).

[21] S. Takeno and M. Goda, Prog. Theor. Phys. 45, 331 (1971). 